\title{
What Are the Foods We Need to Avoid in Acute COVID-19 Infection?
}

\author{
Huang Wei Ling* \\ Medical Acupuncture and Pain Management Clinic, Brazil
}

\section{Editorial}

The theme that the author will discuss in this editorial article on which foods should be avoided in the acute process of infection by COVID-19, is not a topic much talked about in infectious diseases congresses and also in nutrition congresses, in which the author has participated, but this theme is very important for the knowledge of the doctors today, because it is essential to know how to know which foods the patient can ingest and which food the patient need to avoid, that can contribute to better evolution of the COVID-19 infection process. ${ }^{1}$

The author is an infectious disease specialist by Londrina State University in Brazil (1995) and after her specialization, she obtained a specialization on parenteral and enteral nutrition by the Brazilian Society of Enteral and Parenteral Nutrition and has been working with traditional Chinese medicine and Chinese dietary therapies for the last 24 years of work. ${ }^{2}$

What is the difference between Western medicine-based nutritional therapy and Chinese dietary therapy? Western dietary therapy consists of evaluating the food components of each food, an amount of carbohydrates, proteins, lipids, vitamins, etc. and in Chinese dietary therapy, foods are classified according to their energy value, as they are divided into Cold, Neutral, Warm and Hot foods. ${ }^{3}$

To understand how to choose the type of food to use in COVID-19 infection, the physician needs to understand the energy alterations that is occurring in the patients during the acute infection of SARS-CoV-2 invasion. This classification of foods according to their energy properties are important because the physician needs to understand what is happening in the energy point of view, to do no harm even more the disequilibrium of the internal energy of the patient and leading to a worse evolution during the treatment process. ${ }^{4}$ To understand which kind of food we need to avoid in this infection, the author conducted a study of the energy pattern presented by 409 patients files, taken from a total of 1000 patients analyzed between 2015 and 2020, testing their energy presented in the chakras' energy centers. ${ }^{4}$

Chakras' are energy concentrations that govern the functioning of all internal organs and external sensory organs. Measuring the energy of the chakras', the author indirectly intends to measure the energy of the five internal massive organs (Spleen, Lung, Kidney, Liver and Heart), responsible for the production of Yin, Yang, Qi and Blood, which are important for the maintenance of the individual's health. ${ }^{5}$ The author will demonstrate which are the energy alterations in each phase of infection and show the type of food we should avoid at each stage of the infection, to do not lead to more complication of the evolution of these patients, according to the energy imbalance presented by each patient, in a certain stage of the disease. ${ }^{4}$

According to a study written by Huang (2021) entitled Energy Alterations and Chakras' Energy Deficiencies and Propensity to SARS-CoV-2 Infection, the author concluded that more than $90 \%$ of her patients have lack of energy in the five internal massive organs (Heart, Spleen, Lung, Kidney, Liver) of the Five Elements theory of traditional Chinese medicine reasoning, that command the produc-

\begin{tabular}{|l|l|}
\hline Quick Response Code: & *Corresponding author: Huang Wei Ling, Infectious Diseases, General Practice, Nutrition, \\
Acupuncture and Pain Management, Medical Acupuncture and Pain Management Clinic, Rua \\
Homero Pacheco Alves, 1929, Franca, São Paulo, Brazil \\
Received: 01 September, 2021 \\
Citation: Wei Ling H. What Are the Foods We Need to Avoid in Acute COVID-19 Infection? Glob \\
Nutri Heal Food Sci J. 2021;1(1):1-4. DOI: 10.53902/GNHFSJ.2021.01.000501
\end{tabular}


tion of Yin, Yang, Qi and Blood for the maintenance of life. ${ }^{4}$

In traditional Chinese medicine, all external sensorial organs are interconnected and one organ depends on energy from another organ to maintain its functions. ${ }^{3}$ To understand the type of food we should avoid in acute SARS-CoV-2 infection, we must keep in mind to know how to understand the evolution of COVID-19 in terms of energy and what are the energy imbalances involved in each phase, in order to understand which type of food should be ingested and how to prepare them so as not to worsen the patient's evolution. ${ }^{4}$

COVID-19 infection is caused by the invasion of external pathogenic factor, according to traditional Chinese medicine, and all external pathogens such as Wind, Cold, Heat, Humidity and Dryness, are the factors that may be entering along with virus and causing the symptomatology, and the symptomatology presented by each patient will depend on the type of energy imbalances that each patient had before the acquisition of infection and on the type of external pathogenic factor that is entering inside the patients' body , together with the virus. ${ }^{4}$

The most common and classic external pathogenic factors that cause runny nose and sore throat are the invasion of Cold and Wind and the author will base on these two factors mainly to guide which foods the patients have to eat, during their infection. ${ }^{4}$ The foods that the patient has to avoid at this first stage are Cold energy foods, which are almost all raw fruits and vegetables and only eat cooked, baked or dried foods with the exception of papaya, apple and lemon, which are considered neutral foods. ${ }^{4}$

The water cannot be consumed Cold and at the room temperature it is already considered Cold energy, therefore it will aggravate the entry of external pathogens if the patient is consuming the Cold water or water in the room temperature. Therefore, drinking water at this stage has to be boiled (to transform the Cold into neutral energy) and taken it warm at a temperature of about 35 degrees in internal body's temperature. ${ }^{4}$

In this phase, it is very important to prevent the entry of an external pathogenic factor, giving correct nutrition. It is also very important that the patient is adequately covered, so that the penetration of the external pathogenic factor does not occur. ${ }^{4}$ In the article written by Harrison et al. (2020) entitled Mechanisms of SARS-CoV-2 Transmission and Pathogenesis, they are saying that SARS-CoV-2 infections presents with flu-like symptoms and can progress to systemic inflammation and many organs' insufficiencies or failure. ${ }^{6}$

If the patient goes to phase two and three of this infection, these two phases are characterized by the internalization of these external pathogenic factors and deepening inside the body, invading the internal organs, when there is no elimination of this external pathogenic factor in phase one. ${ }^{4}$
In this phase 2, there is the manifestation of inflammatory process but according to the article written by Huang (2021) entitled Energy Alterations and Chakras' Energy Deficiencies and Propensity to SARS-CoV-2 Infection, studying the type of patient that we are treating and their energy imbalances of Yin, Yang, Qi ,Blood, and formation of internal Heat, prior of COVID-19 infection, the author is explaining that the inflammatory process in this phase is characterized by the formation of internal Heat that some patients could have prior to their infections, due to energy deficiency that the majority of the population could be presenting nowadays. As showed by the author, when the patient has energy deficiency, there is formation of internal Heat, that is responsible for the formation of inflammatory process seeing in the laboratory exams, and that could worse with the type of food that the physician will prescribe and depending on the type of medication that the patient will receive. ${ }^{4}$

This inflammatory process, in this phase 2 and 3 are very well explained by the author in the articles written by Huang (2018) entitled Why Do Patients Still Catch Hospital Infections Despite the Practice of Infection Prevention and Control Programs? and in the second article also written by Huang (2019) entitled How Can We Treat Recurrent Herpes Virus Infection Without the Use of Antiviral Drugs?.7,8

At this stage, we must give foods that do not cause more internal Heat formation and also give foods to balance the internal five massive organs (Liver, Heart, Spleen, Lung and Kidney) and avoid more energy depletion to harm the absorption of nutrient, due to erroneous food intake. ${ }^{4}$ The input of energy to be distributed to the rest of the body is carried out by the Spleen-pancreas meridian (fifth chakra), which is deficient in $97 \%$ of its patients in the study written by Huang (2021) entitled Are We Vaccinating Immunocompetent or Immunocompromised People for COVID 19?

To improve the absorption of nutrients in these patients, it is necessary to avoid the ingestion of all dairy products such as cheese, curd, yogurt, butter and all foods that are made from cow's milk. Also, it is necessary to avoid Cold water, raw foods and sweets. ${ }^{7}$ Patients on enteral nutritional support, should receive these cow's milk-free diets. These patients undergoing enteral nutrition therapy, should receive warm water between diets to tone the energy from the Spleen-pancreas and this water has to be around 32 to 35 degrees Celsius, to tone and balance this meridian, which is deficient in most patients ( $97 \%$ of the patients studied by the author). ${ }^{9}$ Enteral diets should never be given Cold to the patient, but at an internal body temperature around 35 degrees. ${ }^{7}$

To tone the Lung's energy, foods rich in green flowers and leaves should be given to the patient. Foods such as broccoli, cauliflower and green leaves improve Lung's energy and should always be prepared steamed, cooked, roasted or sautéed, instead of giving it raw 
to avoid unbalancing the energy of the Spleen-pancreas, causing a greater damage in the absorption of nutrients. ${ }^{7}$

To improve Kidney's energy, responsible for the production of Yin and Yang energies, we should guide the patient to avoid ingestion of coffee, mate tea and soft drinks and increase fluid and water intake, around 1 liter for every $25 \mathrm{~kg}$ of weight per day, and avoid natural fruit juices, which have Cold energy, with the exception of lemon, papaya and apple, which have neutral energy. This is very important to know because it is very frequent to see patient drinking a lot of orange juice to improve their vitamin $\mathrm{C}$, but they are worsening the entrance of external pathogenic factor in this stage, worsening the evolution of SARS-COV-2 infection. ${ }^{7}$ To reduce the production of internal Heat, thus reducing the inflammatory process that is very characteristic of these phases, we should guide the patient to avoid ingesting chocolate, fried foods, coconut, honey, eggs, melted cheese, spice foods and alcoholic beverages. ${ }^{4,7}$

At this stage, due to the production of internal Fire (secondary to energy deficiencies), being very intense in these patients (that the energy imbalance responsible for the inflammatory process), vigorous hydration in these patients is recommended, due to the consumption of body's fluid, by this internal Fire. The observation of the mouth mucosa, skin turgor and urine density and urine color should guide the physician to adequately hydration of these patients, so that they can evolve in a way that has no chance of evolving to pre-renal renal failure. Therefore, lack of adequate hydration can lead to Kidney failure and be misinterpreted as caused by viral infection. 4 ,7

Patients who have a history of routine ingestion of cow's milk and Cold water in their history, may have a greater chance of progressing to renal failure and, therefore, it is important to remove the milk during this phase and introduce warm water, in addition to avoiding raw foods and they are factors that increase the chance of developing renal failure, according to traditional Chinese medicine, when there is an imbalance of the energy of the Spleen-pancreas, through the Control cycle of the Five Elements theory, of traditional Chinese medicine. ${ }^{10}$

In stage 3 of SARS-CoV-2 infection, it is characterized by the worsening of energy imbalance, with the formation of intense internal Heat and characterized by hemorrhages, irritability, and reddish spots on the skin, among many other symptoms. ${ }^{4}$ It is very important at this stage to keep the foods recommended in phase 2 and avoid drinking Cold water and all the foods recommended to avoid in phase 1 and 2 must keep in the mind (such as ingestion of Cold water and raw foods should still have avoided in these phase). ${ }^{4}$

It is also very important to guide the nursing staff to adequately cover the patient during all stages of SARS-CoV-2 infection. If the patient is being treated in a country with a Hot climate, he should at least be covered with a thin sheet. If the patient is being treated in a country with a Cold climate, he should be advised to be covered with a blanket to prevent the entry of external pathogenic factor, especially in the upper part of the chest, where Cold and Wind normally enter, causing more energy imbalances with more formation of internal Heat, generating more inflammatory process, as Huang (2021) wrote in the article Energy Alterations and Chakras' Energy Deficiencies and Propensity to SARS-CoV-2 Infection. ${ }^{4}$

Proper dietary guidelines are just as important as the medications prescribed for the patient during this infection. According to Hippocrates, make your food your medicine and your medicine your food. ${ }^{11}$ This article was written based on the theories of traditional Chinese medicine and Chinese dietary nutrition therapy, as said by Hippocrates, we should consider older medicines before current medical practice. For this reason, the author chose to write about this topic of which kind of food that the physician needs to orientate the patient to avoid in the acute phase of SARS-COV-2 infection, because the ingestion of wrong and improperly prepared foods, can further unbalance internal energy, leading to more complications even fatal evolution of patient in treatment of COVID-19 infection. ${ }^{4,11}$

The incorrect use of food can further unbalance the energy alterations that the patients could have existed, before the patient acquired this infection. And for more chance of having a more favorable evolution and thus, having fewer complications and less need to use medication, which nowadays is a factor that contributes to worsen the patient's evolution, according to the article written by Huang (2021) entitled Is the Medication used in Intubation of Patients with Covid-19 Affecting the Outcome of the Patient's Treatment? In this article, the author is showing that all types of medications in high concentrations today, including medications for fever, anti-inflammatory, antibiotics, anti-coagulant, etc, can induce more energy deficiency, worsening the evolution of patients with SARSCoV-2 infection, based on Arndt Shultz Law. ${ }^{12}$

So, for the treatment of patients with COVID-19, we need to understand the whole process involved in this infection, in the energy point of view, integrating the reasoning od Western medicine with traditional Chinese medicine, and the type of food that the physician need to orientate the patient to avoid is very important in all stages of the infection, to do not worsen the evolution of patient that is treating this infection, that is normally said caused by the virus, but if we understand all the alterations in the human body, prior to this infections, we will know which kind of food and medication we need to avoid to do no harm even more the internal energy of this kinds of patients, that is very low, as the author demonstrated in the study Energy Alterations and Chakras' Energy Deficiencies and Propensity to SARS-CoV-2 Infection. ${ }^{4}$ 
As said by Hippocrates, we need to treat the patient and not the

$$
\text { disease. }{ }^{11}
$$

\section{Acknowledgments}

None.

\section{Funding}

This work had no funding of any kind.

\section{Conflicts of Interest}

Author declares that there is no conflict of interest.

\section{References}

1. Huang Wei Ling. Can We Treat Children with Chronic Respiratory Tract Infections Without Using Antibiotics?. Pediatr Res Child Health. 2020;3(2)

2. Ling HW. What have behind in all kinds of infections that we need to know?. Journal of Investigative Oncology. 2021;1(1):18-21.

3. Huang Wei Ling, Why Are Diabetic Patients Still Having Hyperglycemia Despite Diet Regulation, Antiglycemic Medication and Insulin?. Int J Diabetes Metab Disord. 2019;4(2):1-14.

4. Huang Wei Ling. Energy Alterations and Chakras' Energy Deficiencies and Propensity to SARS-CoV-2 Infection. Acta Scientific Microbiology. 2021;4(4):167-196.

5. Huang Wei Ling. The Importance of Treating Energy Imbalances and Chakras Replenishment for Prevention and Treatment Chakras Replenishment for Prevention and Treatment of Cancer. Advances in Cancer Research \& Clinical Imaging. 2020;3(1):1-10.

6. Harrison A. Mechanisms of SARS-CoV-2 Transmission and Pathogenesis. Trends Immunol. 2020;41(12):1100-1115.

7. Huang Wei Ling. Why Do Patients Still Catch Hospital Infections Despite the Practice of Infection Prevention and Control Programs?. Acta Scientific Microbiol. 2018;1(4):34-43.

8. Huang Wei Ling. How Can We Treat Recurrent Herpes Virus Infection Without the Use of Antiviral Drugs?. Acta Scientific Medical Sciences. 2019;3(8):152-159.

9. Huang WL. Are We Vaccinating Immunocompetent or Immunocompromised People for COVID 19?. J Vaccines Res Vaccin. 2021;7:018.

10. Huang WL. Energy Alterations and Treatment in Systemic Lupus Erythematosus Patients with Altered Proteinuria. ARC Journal of Nephrology. 2019;4(2):22-32.

11. Craik E. The Hippocratic Corpus: Content and Context. Routledge. 2014: p. 344.

12. Huang Wei Ling. Is the Medication used in Intubation of Patients with Covid-19 Affecting the Outcome of the Patient's Treatment?. Archives of Anesthesiology. 2021;4(1):01-03. 